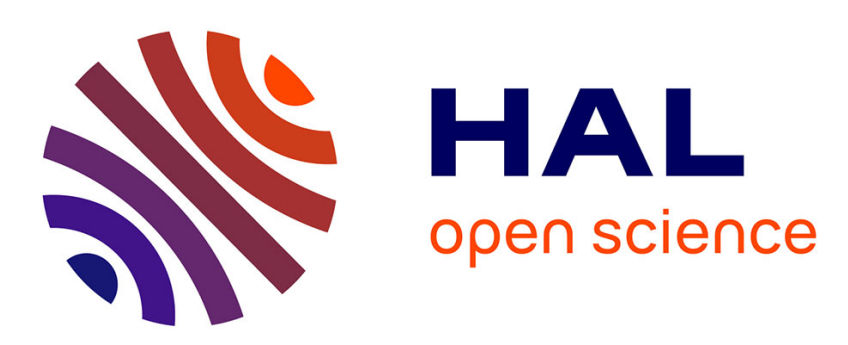

\title{
A simple statistical phenomenological model for cation substitutions in $\mathrm{Nd} 1+\mathrm{xBa} 2-\mathrm{xCu} 3 \mathrm{O} 7-\mathrm{d}+\mathrm{x} / 2$
}

Marcello Gombos, Enrico Varesi, Patrizia Tedesco, Antonio Vecchione, Sandro Pace

\section{- To cite this version:}

Marcello Gombos, Enrico Varesi, Patrizia Tedesco, Antonio Vecchione, Sandro Pace. A simple statistical phenomenological model for cation substitutions in $\mathrm{Nd} 1+\mathrm{xBa} 2-\mathrm{xCu} 3 \mathrm{O} 7-\mathrm{d}+\mathrm{x} / 2$. Philosophical Magazine, 2008, 88 (09), pp.1389-1399. 10.1080/14786430802178079 . hal-00513903

\section{HAL Id: hal-00513903 https://hal.science/hal-00513903}

Submitted on 1 Sep 2010

HAL is a multi-disciplinary open access archive for the deposit and dissemination of scientific research documents, whether they are published or not. The documents may come from teaching and research institutions in France or abroad, or from public or private research centers.
L'archive ouverte pluridisciplinaire HAL, est destinée au dépôt et à la diffusion de documents scientifiques de niveau recherche, publiés ou non, émanant des établissements d'enseignement et de recherche français ou étrangers, des laboratoires publics ou privés. 


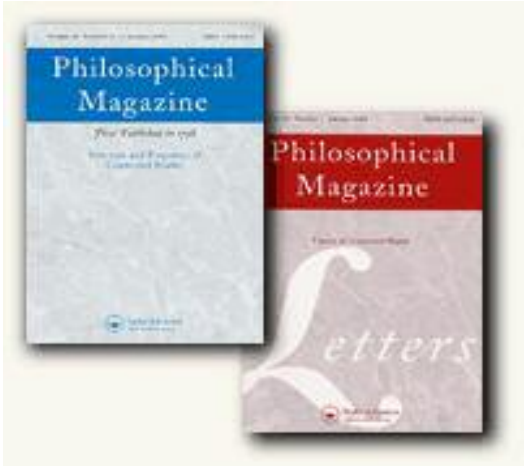

\section{A simple statistical phenomenological model for cation substitutions in Nd1 +xBa2-xCu307-d +x/2}

\begin{tabular}{|r|l|}
\hline Journal: & Philosophical Magazine \& Philosophical Magazine Letters \\
\hline Manuscript ID: & TPHM-07-Nov-0338.R1 \\
\hline Journal Selection: & Philosophical Magazine \\
\hline Date Submitted by the \\
Author: & 26-Mar-2008 \\
\hline Complete List of Authors: & $\begin{array}{l}\text { Gombos, Marcello; CNR-INFM, Laboratorio Regionale SuperMat } \\
\text { Varesi, Enrico; StMicroelectronics, central R\&D-Agrate } \\
\text { Tedesco, Patrizia; Università di Salerno, Dip. di Fisica } \\
\text { "E.R.Caianiello" } \\
\text { Vecchione, Antonio; CNR-INFM, Laboratorio Regionale SuperMat } \\
\text { Pace, Sandro; CNR-INFM, Laboratorio Regionale SuperMat }\end{array}$ \\
\hline Keywords: & cuprate superconductors, phase diagrams, solid solutions \\
\hline Keywords (user supplied): & NdBaCuO \\
\hline
\end{tabular}

\section{S) ScholaroNE \\ Manuscript Central}


Philosophical Magazine

Vol. X, No. X, Month 200X, 000-000

\title{
A simple statistical phenomenological model for cation
}

\section{substitutions in $\mathrm{Nd}_{1+\mathrm{x}} \mathrm{Ba}_{2-\mathrm{x}} \mathrm{Cu}_{3} \mathrm{O}_{7-\delta+\mathrm{x} / 2}$}

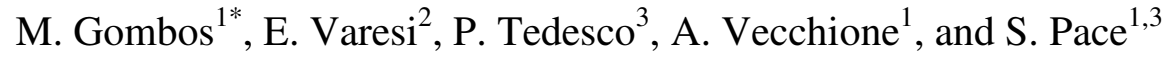 \\ ${ }^{1}$ I.N.F.M. Supermat, Via Salvador Allende, I-84081 Baronissi (SA) Italia. \\ ${ }^{2}$ S.T.Microelectronics, central R\&D-Agrate, Via Olivetti 2, I-20041 Agrate Brianza (MI), Italia. \\ ${ }^{3}$ Università degli Studi di Salerno, Dipartimento di Fisica “E.R. Caianiello”, Via Salvador \\ Allende, I-84081 Baronissi (SA) Italia.
}

(Received 23th November 2007, Modified 26th March 2008)

\begin{abstract}
In this article we present the first results of a quite simple phenomenological model we have developed to simulate the cationic substitutions in $\mathrm{Nd}_{1+\mathrm{x}} \mathrm{Ba}_{2-\mathrm{x}} \mathrm{Cu}_{3} \mathrm{O}_{7+\mathrm{x} / 2-\delta}(\mathrm{Nd} 123)$. Although elementary concepts from Statistical Mechanics had been used in it, significant results have been obtained, as the reconstruction of the substitution region limits and their dependence on temperature. Particularly interesting is the prediction of strong temperature dependence for the minimum of the substitution parameter $x$.
\end{abstract}

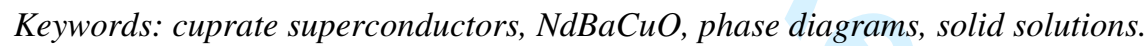

\footnotetext{
*Corresponding author. Email: gombos@sa.infn.it
} 


\section{Introduction}

It is well known that cuprate superconductors $\mathrm{RE} 123\left(\mathrm{REBa}_{2} \mathrm{Cu}_{3} \mathrm{O}_{7-\delta}, \mathrm{RE}=\mathrm{Y}\right.$ or rare earth), belonging to the $\mathrm{RE}-\mathrm{Ba}-\mathrm{Cu}-\mathrm{O}$ system, present plenty of interesting characteristics. In particular $\mathrm{Nd} 123$ shows high values of critical temperature $T_{c}$ and high critical current density $J_{c}$ in presence of large magnetic fields. Yoo and McCallum [1] observed that the rates among stoichiometric quantities of the constituting elements were variable, leading to the formula:

$$
\operatorname{Nd} 123(x)=\mathrm{Nd}_{1+\mathrm{x}} \mathrm{Ba}_{2-\mathrm{x}} \mathrm{Cu}_{3} \mathrm{O}_{\mathrm{z} 1}
$$

where $x$ is the variable substitution parameter and $\mathrm{z}_{1}=7-\delta+x / 2$ [2] since, to preserve ion charges balancing, oxygen ions number slightly varies in dependence to $x$, progressively filling $\mathrm{O}(5)$ sites. Formula Eq.1 corresponds to hypothesising that the allowed variations in RE123 stoichiometry do not change the general structure of crystal lattice. In fact the number of $\mathrm{Cu}$ ions is supposed constant, and also constant is the sum of the stoichiometric coefficients of $\mathrm{Nd}$ and $\mathrm{Ba}$ ions, so that each variation in $\mathrm{Nd}$ content is associated to a corresponding opposite variation in $\mathrm{Ba}$ content. This means that these variations are correlated to substitutions of supernumerary $\mathrm{Nd}^{3+}$ ions in $\mathrm{Ba}^{2+}$ sites (Fig.1a), allowed by the small difference between $\mathrm{Nd}^{3+}$ and $\mathrm{Ba}^{2+}$ ionic radii (less then $20 \%$ ). On the contrary, $\mathrm{Y}^{3+}$ is decidedly smaller than $\mathrm{Ba}^{2+}$, so that there are not Ba-Y substitutions in $\mathrm{Y} 123$.

By increasing $x$, a severe worsening in superconducting properties [3, 4] of $\mathrm{Nd} 123$ has been observed, so that for $x>0.4$, a total disappearance of superconductivity occurs. On the other side, the substituted phases seem to play a role in magnetic flux pinning [5, 6], determining for high magnetic fields critical current densities higher than Y123 ones [7].

Substitutions are experimentally favoured by high temperature treatments and, due to charge balancing, by high oxygen pressure. In fact Nd123 powders characterised by the minimum substitutions have been synthesised performing fabrication processes in reduced oxygen atmosphere $\left(\mathrm{P}_{\mathrm{O} 2} \approx 0.01 \mathrm{Atm}[7]\right)$ followed by low temperature oxygenation processes.

Some characteristics of $\mathrm{Nd}-\mathrm{Ba}-\mathrm{Cu}-\mathrm{O}$ system are summarised in its $890^{\circ} \mathrm{C}$ ternary phase diagram (Fig.2). In the diagram appear two ionically substituted phases: $\mathrm{Nd} 123(x)$ and $\mathrm{Nd} 422(z)$, where $\mathrm{Nd} 422(z)$ is [5]:

$$
\mathrm{Nd} 422(z)=\mathrm{Nd}_{4-2 z} \mathrm{Ba}_{2+2 z} \mathrm{Cu}_{2-z} \mathrm{O}_{10-2 \mathrm{z}}
$$

Until now, the exact variation range of the substitution parameters $x$ and $z$ hasn't been determined. In literature the reported [8,9] values for the extremes of the variation interval of $x$ are $0<x_{\min }<0.04$ and $0.6<x_{\max }<1$. Nd123 with zero substitution seems to be, in fact, unstable with respect to substituted phases. Moreover, $x_{\max }$ has shown a dependence on $\mathrm{T}$ [10] and on $\mathrm{P}_{\mathrm{O} 2}$ [7]. In the following we will use for the values of the extremes, data taken by Goodilin et al. [10] and (for $\mathrm{T}=$ $890^{\circ} \mathrm{C}$ ) by Yoo and McCallum [1], $\left(\mathrm{x}_{\min }=0.04\right.$ and $\left.\mathrm{x}_{\max }=0.6\right)$.

For $\mathrm{Nd} 422$ the experimentally determined extremes at $890^{\circ} \mathrm{C}$ [11] are $-0.12<z<0.13$ (the formula used for $\operatorname{Nd} 422(z)$ in [11] is different from Eq.2, although equivalent to it [12], so we have converted the results).

The appearance of substituted phases has been observed in all processes involving Nd123, such as powder synthesis, film deposition, sintering, melt texturing etc. Particularly complex is the case of melt texturing techniques, in which $\mathrm{Nd} 422(z)$ is added to $\mathrm{Nd} 123$ powders. In this case, they react following the reaction [12]:

$$
a_{1} \operatorname{Nd} 123\left(x_{1}\right)+b_{1} \operatorname{Nd} 422\left(z_{1}\right) \Rightarrow a_{2} \operatorname{Nd} 123\left(x_{2}\right)+b_{2} \operatorname{Nd} 422\left(z_{2}\right)
$$


\[ a_{2}=a_{1} \frac{5-x_{1}}{5-x_{2}} \text { and } \mathrm{b}_{2}=\mathrm{b}_{1} \text { with the condition } b_{1}\left(z_{1}-z_{2}\right)=3 a_{1} \frac{x_{1}-x_{2}}{5-x_{2}} \text {. Experimentally it is } \]
where
always $x_{2} \geq x_{1}$, indicating the existence of a preferred direction determined by the thermodynamics of
Nd-Ba-Cu-O system.

In this work our purpose has been then to develop a simple and realistic statistical model for Nd123 solid solution. The main behaviours we will try to justify are:

1) the tendency of our system $\mathrm{Nd} 123(x)$ to increase its $\mathrm{Nd}$ content in the solubility region,

2) the increase of maximum $\mathrm{Nd}$ substitution parameter $x_{\max }$ as a function of temperature $\mathrm{T}$,

$3)$ the instability of non substituted $\mathrm{Nd} 123$ with respect to slightly substituted $\operatorname{Nd} 123\left(x_{\min }\right)$ phase.

\section{The model}

\subsection{The solubility region}

In $\mathrm{Nd}_{1+\mathrm{x}} \mathrm{Ba}_{2-\mathrm{x}} \mathrm{Cu}_{3} \mathrm{O}_{\mathrm{z} 1}$ system, $\mathrm{Nd}^{3+}$ and $\mathrm{Ba}^{2+}$ number varies, so we should use the Grand Canonical Ensemble formalism. To simplify our calculations we can close the system, by writing it as a decomposition reaction, and use the Canonical Ensemble formalism. We can analyse then the solubility region of the system by calculating its Gibbs free energy $\mathrm{G}$ as a function of $\mathrm{Nd}$ substitutions. The reaction that closes the system is:

$$
\mathrm{Nd} 123(0)+x \mathrm{Nd}^{3+} \Rightarrow \mathrm{Nd} 123(x)+x \mathrm{Ba}^{2+}
$$

This is a net reaction made by simplifying an original one like:

$$
\mathrm{Nd} 123(0)+\left(\mathrm{N}_{\mathrm{Nd}}\right) \mathrm{NdO}_{1.5} \Rightarrow \mathrm{Nd} 123(x)+\left(\mathrm{N}_{\mathrm{Nd}}-x\right) \mathrm{NdO}_{1.5}+x \mathrm{BaO}+(x / 4) \mathrm{O}_{2}
$$

Eq.5 is the simplest reaction compatible with Eq.4, in fact $\mathrm{Nd}^{3+}$ ions come from $\mathrm{Nd}_{2} \mathrm{O}_{3}$ and $\mathrm{Ba}^{2+}$ is only present in the products as $\mathrm{BaO}$ (that in air further reacts with $\mathrm{CO}_{2}$ to form $\mathrm{BaCO}_{3}$ ). Eq.4 would be also compatible with Eq.3, but the statistical treatment of Eq.3 would need also a model for $\mathrm{Nd} 422(\mathrm{z})$ that is beyond the purpose of our discussion. In our derivation we will also neglect the effect of atmospheric oxygen and gas evolution $\left(\mathrm{O}_{2}\right.$ or $\left.\mathrm{CO}_{2}\right)$, because the data we have used are homogeneous with respect to these experimental parameters.

The minimum of $\mathrm{G}$, corresponding to the substitution parameter $\mathrm{x}_{\max }$, will give the stable state of the system in the presence of an external source of $\mathrm{Nd}$ ions. Any further $\mathrm{Nd}$ addition would be energetically unfavourable, inducing the phase to decompose into $\mathrm{Nd} 123\left(x_{\max }\right)+\mathrm{NdO}_{1.5}$ again. In this way the Gibbs energy minimum gives the maximum substitution state.

$\mathrm{G}$ is constituted by an entropy term and an energy one. Since entropy is written in terms of the configurations number $\Omega$ as $S=k_{B} \ln (\Omega)$, its easy to calculate the difference between entropy of the left and of the right side terms of the substituted phase in Eq.4. Below its peritectic temperature Tp, $\operatorname{Nd} 123(x)$ is a solid solution in which, as shown in Fig.1b, for $N$ formula units, $N x$ supernumerary $\mathrm{Nd}^{3+}$ are dissolved in a matrix constituted by $2 \mathrm{~N} \mathrm{Ba}^{2+}$ sites .

In this case entropy is:

$$
\mathrm{S}_{\mathrm{Nd}}(\mathrm{x})=-\mathrm{k}_{\mathrm{B}} N(x \ln (x)+(2-x) \ln (2-x)-2 \ln (2))
$$

The factor $-2 \ln (2)$ allows to have $\mathrm{S}_{\mathrm{Nd}}=0$ for $x=0$.

The variation of internal energy of $\mathrm{Nd} 123(\mathrm{x})$ is determined, in a zero order approximation, by the interaction of supernumerary neodymium ions with the electric field, supposed fixed and independent on $x$, generated in the Ba sites by the (unperturbed) crystal lattice ions. In this way the 
M. Gombos et al.

energy variations $\Delta \mathrm{H}_{0 \mathrm{Nd}}$, due to each $\mathrm{Nd}$ ion, is given by the product of the difference between $\mathrm{Nd}$ and $\mathrm{Ba}$ ionic charges, multiplied by an effective electrostatic potential $\mathrm{U}_{0}$. This potential accounts also of lattice deformations due to differences in ionic radii, energy variation of electrons system, possible variations in oxygen contents, etc [13]. Following Eq.5, for each $\mathrm{Nd}$ substitution another energy term proportional to $N x$, due to chemical potentials difference (we write as $\mu_{\mathrm{Ba}}-\mu_{\mathrm{Nd}}=\mathrm{q}_{\mathrm{e}} \mu$, where we use here $\mathrm{q}_{\mathrm{e}}$, the (positive) electronic charge, to simplify calculations) between absorbed $\mathrm{NdO}_{1.5}$ and produced $\mathrm{BaO}$, must also be added. To simplify notation we will absorb both the electrostatic cell potential and this last chemical potential into a linear potential term $U=U_{0}+\mu$. To complete the picture, $\mathrm{NdO}_{1.5}$ and $\mathrm{BaO}$ contributions to entropy are both equal to $\mathrm{k}_{\mathrm{B}} N x$ and cancel each other. In this approximation $\mathrm{G}$ can be written as:

$$
\mathrm{G}=N\left(x \mathrm{q}_{\mathrm{e}} \mathrm{U}+\mathrm{k}_{\mathrm{B}} \mathrm{T}(x \ln (x)+(2-x) \ln (2-x)-2 \ln (2))\right)
$$

The minimum of Eq.7 with respect to substitution parameter $\mathrm{x}$ gives the stability curve $\mathrm{T}(\mathrm{x})$ which represents the stable substitution $\mathrm{x}$ at each temperature:

$$
\mathrm{T}=\frac{q_{e} \mathrm{U}}{k_{B}(\ln (2-x)-\ln (x))}
$$

Unfortunately, whatever $\mathrm{U}$ (even using the value $\mathrm{U}=1,1910^{-2} \mathrm{~V}$, obtained from the least square best fit method applied on the experimental data $[1,10])$, the zero order approximation solution Eq.8 gives temperature dependencies that do not fit the experimental data reported in [10] and [1] (Fig.3). For this reason it is necessary to introduce an interaction among the supernumerary $\mathrm{Nd}$ ions present in $\mathrm{Nd} 123(x)$. To simplify our task we will suppose each ion to interact only with nearest ions that, in a first hypothesis, are homogeneously distributed inside the lattice. Considering the total crystal volume $N \cdot a \cdot b \cdot c$ ( $a, b$ and $c$ are the lattice parameters) and the occupied sites number $N x$, the volume allowed to each supernumerary $\mathrm{Nd}$ ion is: $V_{F}=a b c / x$, the nearest ions mean distance is then of the order:

$$
r \approx V_{F}^{\frac{1}{3}}=\left(\frac{a b c}{x}\right)^{\frac{1}{3}} .
$$

By supposing an electrostatic interaction among $\mathrm{Nd}$ ions, the corrective term will be then:

$$
\Delta H_{1 N d}^{T o t}=N x \Delta H_{1 N d}=N x \frac{K n\left(q_{N d}-q_{B a}\right)^{2}}{r}=N x \frac{K n q_{e}^{2}}{(a b c)^{\frac{1}{3}}} x^{\frac{1}{3}}
$$

where $\mathrm{K}$ is the electrostatic constant. The $n$ coefficient is a constant that may depend on the nearest ions number, on a proportionality geometrical factor between $r$ and $V_{F}^{1 / 3}$ (and so it should be related to the Madelung constant of the crystal), and may also account for the effective screening of the charge (or any other cause affecting the Nd ions interaction). In an ideal case, where no screening or polarisation are considered, this number should equal the number of nearest substituted ions (that is usually between 2 and 8). So we will be able to estimate the relevance of the screening by comparing the results we will obtain for $n$ and the number of nearest neighbours predicted in our model. A brute approximation, made by integrating the substituted ions density over a $r$ radium sphere and subtracting 1 (the central ion) would give $n \approx 3.2$, while geometrical reasoning would give the slightly larger value $n \approx 5$, so the expected value of the "screening free" $n$ parameter, for our model, should be near to these ones.

The interaction determines the specific power dependence of $\Delta \mathrm{H}_{1 \mathrm{Nd}}$ on $x$, any interaction with different power law from the hypothesised electrostatic one would lead to a different behaviour of the solution, that in principle may fit the experimental data better or worse than our one. The goodness of our choice will be then measured by how good it will fit the actual behaviour of the 
system.

To simplify our notation we write:

$$
\alpha=\frac{K n q_{e}}{(a b c)^{\frac{1}{3}}} \approx 2.56 n \quad \text { Volts }
$$

so that the Gibbs energy becomes:

$$
\mathrm{G}=N\left(\mathrm{q}_{\mathrm{e}}\left(\mathrm{U} x+\alpha x^{4 / 3}\right)+\mathrm{k}_{\mathrm{B}} \mathrm{T}(x \ln (x)+(2-x) \ln (2-x)-\ln (4))\right)
$$

the condition $\partial \mathrm{G} / \partial \mathrm{x}=0$ gives:

$$
\mathrm{T}=-\frac{q_{e}}{k_{B}} \frac{\left(\mathrm{U}+\frac{4}{3} \alpha x^{\frac{1}{3}}\right)}{\ln \left(\frac{x}{2-x}\right)}
$$

This function is defined for $x \in[0,2]$. It presents a singularity in $x=1$, then this value may be reached only for $\mathrm{T}=\infty$, and two zeros (two essential singularities appearing as zeroes on real axis) at $x=0$ and $x=2$. For $\mathrm{T} \geq 0$ this function assumes, then, two different values in the intervals $x \in[0,1[$ and $x \in] 1,2]$ corresponding to two extremes of Eq.12. To ensure that the value in the interval $x \in[0,1[$ corresponds to a minimum of Eq.12 we must impose $\alpha>-0.75 \mathrm{U}$.

The parameter choice that more accurately simulates the experimental behaviour is $U>0$ and $\alpha<0$. This means that the interaction potential is, in contrast with intuitive reasoning, attractive suggesting a high relevance of screening and polarising effects in our simple electrostatic picture. Moreover, the attractiveness of the interaction potential may cause local clustering of the supernumerary $\mathrm{Nd}^{3+}$ ions and the appearance of highly substituted phase islands in an almost nonsubstituted phase matrix, the mechanism hypothesised to enhance the pinning in $[5,6]$.

Since a theoretical a priori calculation of $U$ and $\alpha$ is not yet available, we will deduce them by fitting the experimental data of Goodilin et al. [10] plus the datum $x(1163 \mathrm{~K})=0.6$ by YooMcCallum [1]. The papers do not report explicitly error bars on their data, but from the text we can assume $\delta \mathrm{T}=25^{\circ} \mathrm{C}$ and $\delta \mathrm{x}<0.01$ (negligeable for our fit), and use error propagation to obtain the error associated to our parameters. The fit gives $U \approx(5.1 \pm 0.4) \cdot 10^{-1} \mathrm{~V}$ and $\alpha \approx-(3.7 \pm 0.1) \cdot 10^{-1} \mathrm{~V}$ corresponding to $\alpha / \mathrm{U}=-0.74$ and $n \approx-(1.47 \pm 0.04) \cdot 10^{-1}$. The obtained $n$ parameter value is more than an order of magnitude lower than the expected one (about 4, as shown above), so this feature gives us an idea of the relevance of the screening effect.

The stability curve for our results is shown in Fig.3, the accuracy with which the fit approximates the data confirms the correctness of our assumptions.

We observe that at temperatures less then $800 \mathrm{~K}$ the most probable values for the substitutions $x$ are lower than $4 \cdot 10^{-2}$. The experimental stability region at ambient and liquid nitrogen temperatures corresponds to values of substitution parameter quite low, but larger than the expected ones. This discrepancy is probably due to the extreme slowness of the relaxation dynamics at such low temperatures. However such small $x$ values support the necessity, empirically determined, to oxygenate $\mathrm{Nd} 123$ at quite low temperature to avoid substitution [14].

Following the model, at high temperature the formation of highly substituted Nd123 is favoured. This is not in contradiction with the experimental fact that most highly substituted states have the lowest peritectic temperatures and form from the melt at lower temperatures than less substituted ones. In fact the previous calculation refers to the lattice in its integrity and cannot be used to make previsions on melting and molten phases.

Moreover, the model suggests a first justification for the reaction Eq.3, linking the increase of $\mathrm{x}$ with the motion of $\mathrm{Nd} 123$ toward its equilibrium substituted state, supposing that $\mathrm{Nd} 422(z)$ may act 
M. Gombos et al.

as an $\mathrm{Nd}$ reservoir. Anyway it is clear that the effective thermodynamics of Eq. 3 could only be studied by determining, analogously to what done for $\mathrm{Nd} 123(x)$, the complete Gibbs free energy of $\mathrm{Nd} 422(z)$ (that would need more data on the temperature dependence of Nd422 substitution) and minimising the total Gibbs energy of the Nd123-Nd422 system.

As a final observation, we do not explicitly consider pressure variations, so the minimised variable is not a Gibbs free energy but a Helmholz free energy. Anyway, by putting the total pressure to zero (as implicitly done in the derivation) distinctions between energies and enthalpies become irrelevant, and we can identify the two functions. Moreover the oxygen chemical potential is constrained to be the same, in the competing phases in Eq.4, by the minimisation procedure (equivalent to the common tangent construction), and its partial pressure value is a-posteriori fixed by the external constraints due to the fitting procedures. These features practically pin the system to the experimental data pressure parameters, so that the thermodynamic potential behaves as a true Gibbs free energy.

\subsection{Instability of stoichiometric $\mathrm{Nd} 123$}

From the Nd-Ba-Cu-O ternary diagram (Fig.2), we observe that for $x_{0}<x_{\min } \mathrm{Nd} 123\left(x_{0}\right)$ is no longer stable but decomposes in $\mathrm{Nd} 123\left(x_{\min }\right)$ and $\mathrm{BaCuO}_{2}$. The general expression of this reaction is [15]:

$$
\operatorname{Nd} 123\left(x_{0}\right)=a \operatorname{Nd} 123(x)+3 b \mathrm{BaCuO}_{2} .
$$

With $a=\frac{1+x_{0}}{1+x}, b=\frac{x-x_{0}}{1+x}$ and $x=x_{\min }$.

As shown in Fig.1a, $\mathrm{Nd} 123$ and $\mathrm{BaCuO}_{2}$ cells are very similar, with very close values of the cell parameters. Then the formation of $\mathrm{BaCuO}_{2}$ from $\mathrm{Nd} 123$ can be described as the substitution of barium ions in the neodymium sites, leading to three $\mathrm{BaCuO}_{2}$ cell for each barium substituted Nd123.

For $x_{0}=0$, Eq.14 describes the instability of the stoichiometric Nd123. In this case the entropy associated to the substitution of $N x \mathrm{Ba}^{2+}$ ions in $\mathrm{Nd}^{3+}$ sites (that means the formation of $3 N x$ cells of $\mathrm{BaCuO}_{2}$ ) is:

$$
\mathrm{S}_{\mathrm{Ba}}(x)=-\mathrm{k}_{\mathrm{B}} N((1-x) \ln (1-x)+x \ln (x)) .
$$

Since such substitution leads to a corresponding substitution of $N x \mathrm{Nd}^{3+}$ in $\mathrm{Ba}^{2+}$ sites, the total entropy of the system is then:

$$
S_{\text {tot }}(x)=a S_{N d}(x)+b S_{B a}(x)=-k_{B} N\left(x \ln (x)+(2-x) \ln (2-x)-2 \ln (2)+\frac{x}{1+x}((1-x) \ln (1-x)-(2-x) \ln (2-x)+2 \ln (2))\right)
$$

The zero approximation energy variation can be written, using an electrostatic potential $U^{*}$ analogous to the one used in Eq.7:

$$
\Delta \mathrm{H}_{0 B a}^{T o t}(x)=N x \Delta \mathrm{H}_{0 \mathrm{Ba}}=N x\left(q_{B a}-\mathrm{q}_{\mathrm{Nd}}\right) \mathrm{U}^{*}=-N x q_{e} \mathrm{U}^{*}
$$

The total variation $\Delta \mathrm{H}_{0}^{T o t}$ is then (as in the previous derivation, we absorbed the chemical potentials contribution in $U_{\text {and }} \mathrm{U}^{*}$ ):

$$
\Delta \mathrm{H}_{0}^{T o t}=a \Delta \mathrm{H}_{0 N d}^{T o t}(x)+b \Delta \mathrm{H}_{0 B a}^{T o t}(-x)=N x q_{e}\left(\mathrm{U}-\frac{x}{1+x}\left(\mathrm{U}^{*}+\mathrm{U}\right)\right)
$$

By supposing, in first approximation, that lattice effects are the same in $\mathrm{Nd} 123$ and in the produced $\mathrm{BaCuO}_{2}$ the interaction energy term between $\mathrm{Ba}$ ions can be written, analogously to Eq.10: 


$$
\Delta \mathrm{H}_{1 B a}^{T o t}=N x \Delta \mathrm{H}_{1 B a}=N x \frac{\left(K n q_{N d}\left(q_{N d}-q_{B a}\right)\right)}{r}=N x \frac{\left(K n 3 q_{e}^{2}\right)}{(a b c)^{\frac{1}{3}}}=N \alpha q_{e} x^{\frac{4}{3}}
$$

The final expression for the interaction term is then the same as in Eq.12:

$$
\Delta \mathrm{H}_{1}^{T o t}=a \Delta \mathrm{H}_{1 B a}^{T o t}(x)+b \Delta \mathrm{H}_{1 B a}^{T o t}(x)=N \alpha q_{e} x^{\frac{4}{3}}
$$

From Eq.19 and Eq.20 the Gibbs free energy is:

$$
\mathrm{G}=N\left(q_{e} x\left(\mathrm{U}-\frac{x}{1+x}\left(\mathrm{U}^{*}+\mathrm{U}\right)+\alpha x^{\frac{1}{3}}\right)+k_{B} T\left(x \ln (x)+(2-x) \ln (2-x)-\ln (4)+\frac{x}{1+x}((1-x) \ln (1-x)-(2-x) \ln (2-x)+\ln (4))\right)\right)
$$

The minimum of this function determines the $\mathrm{x}_{\min }$ value toward which the system spontaneously tends. By equating $\partial \mathrm{G} / \partial \mathrm{x}$ to zero (that is equivalent to the usual common tangent construction, as shown in appendix A) we obtain for the stability line $\mathrm{T}(\mathrm{x})$ :

$$
\mathrm{T}=\frac{q_{e}}{k_{B}} \frac{\mathrm{U}^{*}-\frac{1}{(1+x)^{2}}\left(\mathrm{U}^{*}+\mathrm{U}\right)-\frac{4}{3} \alpha x^{\frac{1}{3}}}{\ln \left(\frac{x}{1-x}\right)+\frac{1}{(1+x)^{2}} \ln \left(\frac{4(1-x)^{2}}{(2-x)^{3}}\right)}
$$

Since no extensive study exist on the dependence of minimum substitution on temperature (and very few experimental data are available) [15], we have decided to only estimate $\mathrm{U}^{*}$ by imposing that at $\mathrm{T}=890^{\circ} \mathrm{C} \mathrm{x}_{\min }=0,04$ [1], and using for $\mathrm{U}$ and $\alpha$ the values found in the previous chapter. This leads to $\mathrm{U}^{*}=1,24 \mathrm{~V}$. The consequent behaviour of the stability line is shown in Fig.4. In this case it is easy to see that $\mathrm{T}(x)>0$ for every $x>0$ and that $\lim _{\mathrm{x} \rightarrow 0} \mathrm{~T}(x)=0$ This means that, whatever may be the temperature, stoichiometric Nd123 is not stable and a minimal substitution is unavoidable. Moreover the dependence of $\mathrm{x}_{\min }$ on temperature is particularly strong in the region from $600 \mathrm{~K}$ up to $\mathrm{T}_{\mathrm{p}}$ (about $1350 \mathrm{~K}$ ).

The main result is that, even in absence of external ions supply, the displacement of $\mathrm{Ba}^{2+}$ ions in $\mathrm{Nd} 123$ crystal resulting in $\mathrm{BaCuO}_{2}$ formation, and the corresponding existence of a minimum $\mathrm{Nd}$ substitution different from zero are due to the entropy contribution. In the low temperature region, the solid solution curve Eq.22 indicates substitution parameter values smaller than the experimental ones. The reason of the actual difficulty to obtain almost stoichiometric Nd123 at low temperature is probably due to the slowness of system dynamics at low temperature, the observed compounds being non-equilibrium thermodynamic states. In fact at the high temperature, at which Nd123 with higher $x_{\min }$ forms, the dynamics associated to Ba displacements is by far swifter than at lower ones. In this way the process of recovering lower substituted states at lower temperatures may be so slow to be experimentally undetectable.

Another mechanism preventing the system from reaching the lowest $x$ states is the $\mathrm{BaCuO}_{2}$ melting at about $1270 \mathrm{~K}$. This process, at temperatures lower than the Nd123 melting temperature, may subtract barium from the system, preventing the reversibility of substitution as the temperature is reduced.

\section{Conclusions}

In the model proposed in this paper $\mathrm{Nd}$ substitutions in $\mathrm{Ba}$ sites of $\mathrm{Nd} 123$ has been analysed by supposing in the Gibbs potential two energy terms, respectively due to charge difference between 
M. Gombos et al.

$\mathrm{Nd}^{3+}$ and $\mathrm{Ba}^{2+}$ ions and to electrostatic interaction between substituted $\mathrm{Nd}^{3+}$ ions, to which it must be added the entropic contribution related to the disorder induced by the substitutions.

By this approach we reconstructed some of the main features of the substitutions phenomenon. Indeed in the solubility region the model describes the tendency of the system to evolve toward higher substituted states and it reconstructs the dependence on $\mathrm{T}$ of the substitution parameter's maximum value. Moreover, the model reproduces the Nd123 decomposition in Nd-substituted phases plus $\mathrm{BaCuO}_{2}$ and the existence of a minimum substitution level $\mathrm{x}_{\min }$ under which the compound is unstable. In other words in our description the impossibility to obtain Nd123(x) with substitution parameter $\mathrm{x}=0$, doesn't derive from the fabrication process, but it is intrinsically linked to the statistics of substitutions. Finally, in our approach $\mathrm{x}_{\min }$ is found to be a growing function of temperature. Measurements of this dependence are still in progress.

These results are obtained by a best fit of data in literature, which determines two free parameters of the model. The validity of our approach could be tested by direct experimental measurement, or by theoretical estimate, of the substitution and interaction energy terms for $\mathrm{Nd} 123$ which are still not available.

Further theoretical work is in progress to take in account the dependence of both minimum and maximum substitution curves on oxygen partial pressure and to develop a model of the substitutions dynamics. 


\section{References}

[1] S.I. Yoo, and R.W. McCallum, Physica C 210, 147-156, (1993).

[2] P. Yossefov, G.E. Shter, G.M. Reisner, A. Friedman, Y. Yeshurun, G.S. Grader, Physica C 275, 299, (1997).

[3] S.I. Yoo, M.J. Kramer, and R.W. McCallum, IEEE T. Appl. Supercon. 3, 1232-1235, (1993).

[4] S. Li, E.A. Hayri, K.V. Ramanujacharry, M. Greenblatt, Phys. Rev. B 38, 2450-2454, (1988).

[5] M. Murakami, S.I. Yoo, T. Higuchi, N. Sakai, M. Watabiki, N. Koshizuka, S. Tanaka, Physica C 235-240, 2781-2782, (1994).

[6] Yu.D. Tretyakov, E.A. Goodilin, Current Appl. Phys. 3, 377-384, (2003).

[7] S. I. Yoo, N. Sakai, H. Takaichi, T. Higuchi, M. Murakami, Appl. Phys. Lett. 65, 633-635, (1994).

[8] G.A. Costa, M. Ferretti, J. Less-Common Met. 157, 77, (1990).

[9] T.B. Lindemer, E.D. Specht, P.M. Martin et al., Physica C 255, 65, (1995).

[10] E.A. Goodilin, N.N. Oleynikov, E.V. Antipov, R.V. Shpachenko, G.Yu. Popov, V.G. Balakirev, Yu.D. Tretyakov, Physica C 272, 65, (1996).

[11] W. Bieger, G. Krabbes, P. Schatzle, L. Zelenina, U. Wiesner, P. Verges, J. Klosowski, Physica C 257, 46-52, (1996).

[12] M. Gombos, V. Gomis, A. E. Carrillo, A. Vecchione, P. Tedesco, S. Pace, and X. Obradors, Supercond. Sci. Technol. 16, 865, (2003).

[13] V.V. Petrykin, E.A. Goodilin, J. Hester, E.A. Trofimenko, M. Kakihana, N.N. Oleynikov, Yu.D. Tretyakov, Physica C 357-360, 388-392, (2001).

[14] V. Gomis, M. Gombos, A. E. Carrillo, J. Plain, X. Obradors, S. Pace, A. Vecchione, Inst. Phys. Conf. Ser. 167, 83-86, (2000).

[15] Yu.D. Tret'yakov, E.A. Goodilin, Russian J. of Inorganic Chem. 46 suppl. 3, s203-s234, (2001). 


\section{Appendix: Equivalence between the Minimisation and the Common Tangent techniques}

We will demonstrate in this appendix the perfect analogy between our minimisation technique and the common tangent procedure. In fact, given two compounds $\mathrm{A}$ and $\mathrm{B}$ and their respective Gibbs free energies (written in function of the compositional parameter $x$ ) $G_{A}(x)$ and $G_{B}(x)$, what we obtain by the common tangent approach is but the minimum of

$$
\mathrm{G}=\mathrm{a} \mathrm{G}_{\mathrm{A}}(\mathrm{x})+\mathrm{b} \mathrm{G}_{\mathrm{B}}\left(\mathrm{x}_{1}\right)
$$

with the set of conditions (or an equivalent one), due to the fixed initial composition:

$$
\begin{aligned}
& a x+b x_{1}=x_{0} \\
& a+b=1
\end{aligned}
$$

Eq.A.2 means indeed:

$$
\begin{aligned}
& \mathrm{a}=\left(\mathrm{x}_{0}-\mathrm{x}_{1}\right) /\left(\mathrm{x}-\mathrm{x}_{1}\right) \\
& \mathrm{b}=1-\mathrm{a}
\end{aligned}
$$

and consequently

$$
\begin{aligned}
& \frac{\partial a}{\partial x}=-\frac{x_{0}-x_{1}}{\left(x-x_{1}\right)^{2}}=-\frac{a}{\left(x-x_{1}\right)^{2}} \\
& \frac{\partial b}{\partial x}=-\frac{\partial a}{\partial x}
\end{aligned}
$$

We can write then the derivative of the total Gibbs energy of the system as

$$
\frac{\partial G}{\partial x}=\frac{\partial a}{\partial x} G_{A}(x)+a \frac{\partial G_{A}(x)}{\partial x}+\frac{\partial(1-a)}{\partial x} G_{B}\left(x_{1}\right)=-a \frac{G_{A}(x)-G_{B}(x)}{x-x_{1}}+a \frac{\partial G_{A}(x)}{\partial x}
$$

then $\partial \mathrm{G} / \partial \mathrm{x}=0$ implies

$$
\mathrm{G}_{\mathrm{A}}(\mathrm{x})-\mathrm{G}_{\mathrm{B}}\left(\mathrm{x}_{1}\right)=\left(\mathrm{x}-\mathrm{x}_{1}\right) \partial \mathrm{G}_{\mathrm{A}}(\mathrm{x}) / \partial \mathrm{x}
$$

that is

$$
\mathrm{G}_{\mathrm{B}}\left(\mathrm{x}_{1}\right)=\mathrm{G}_{\mathrm{A}}(\mathrm{x})+\left(\mathrm{x}_{1}-\mathrm{x}\right) \partial \mathrm{G}_{\mathrm{A}}(\mathrm{x}) / \partial \mathrm{x}
$$

On the other hand, by minimising $\mathrm{G}$ with respect to $\mathrm{x}_{1}$ we would have obtained the equivalent condition

$$
\mathrm{G}_{\mathrm{A}}(\mathrm{x})=\mathrm{G}_{\mathrm{B}}\left(\mathrm{x}_{1}\right)+\left(\mathrm{x}-\mathrm{x}_{1}\right) \partial \mathrm{G}_{\mathrm{B}}\left(\mathrm{x}_{1}\right) / \partial \mathrm{x}_{1}
$$

The resulting Eq.A.7 and Eq.A.8 are exactly the mathematical expression of the common tangent condition, so, given the dependence of $\mathrm{x}_{1}$ on $\mathrm{x}$ and $\mathrm{x}_{0}$ in Eq.A.1, the minimisation of $\mathrm{G}$ with respect to $\mathrm{x}$ gives an analytical solution for the common tangent procedure. 


\section{Figure Captions}

Fig.1a: Crystal structure of stoichiometric $\mathrm{Nd} 123$ (I), $\mathrm{Nd} 123$ with a substituted $\mathrm{Nd}$ ion (II) and $\mathrm{BaCuO}_{2}$ (III).

Fig.1b: Schematic representation of an $\mathrm{Nd} 123(\mathrm{x})$ with $\mathrm{x} \approx 0,1$ (the section is parallel to $\mathrm{c}$ axis).

Fig.2: Ternary phase diagram of $\mathrm{NdBaCuO}$ system at $890^{\circ} \mathrm{C}, \mathrm{P}_{\mathrm{O} 2}=0.2 \mathrm{~atm}$, with the presence of the stability region of the $\mathrm{Nd} 123(\mathrm{x})$ and $\mathrm{Nd} 422(\mathrm{z})$ solid solutions and of the stability line linking $\mathrm{Nd} 123\left(\mathrm{x}_{\min }\right)$ and $\mathrm{BaCuO}_{2}$ (adapted from [1]). Fig.1: Comparison among the experimental stability curve (diamonds, data taken from [10] and [1]), for Nd123(x) and the best fit curves in the zero order approximation (gray line) and in the first order one (black line). The orizontal line at $1390.16 \mathrm{~K}\left(1117^{\circ} \mathrm{C}\right)$ is the maximum reported value of $\mathrm{Nd} 123$ peritectic temperature $\mathrm{T}_{\mathrm{p}}[10]$, above which our model is no more valid.

Fig.4: Temperature dependence of the minimum substitution parameter $\mathrm{x}$ at equilibrium, starting from stoichiometric Nd123 powders. Also here the orizontal line at $1390.16 \mathrm{~K}\left(1117^{\circ} \mathrm{C}\right)$ indicates the maximum $\mathrm{T}_{\mathrm{p}}[10]$, above which our model is no more valid. 


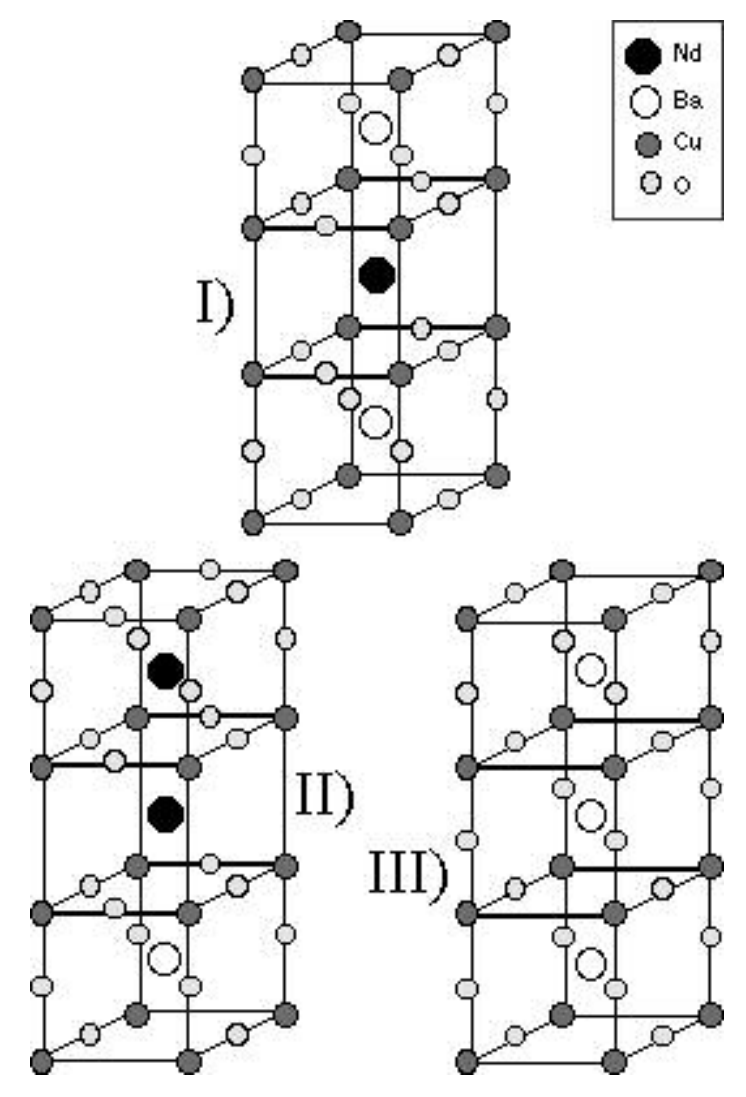

Fig.1a

$93 \times 142 \mathrm{~mm}(72 \times 72$ DPI $)$ 


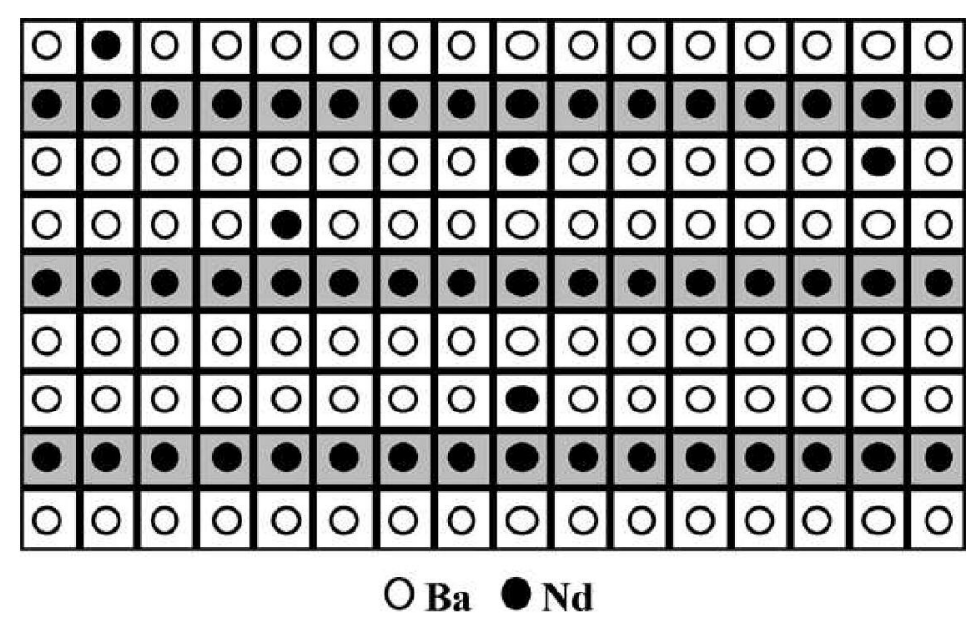

Fig.1b

$184 \times 272 \mathrm{~mm}(600 \times 600 \mathrm{DPI})$ 


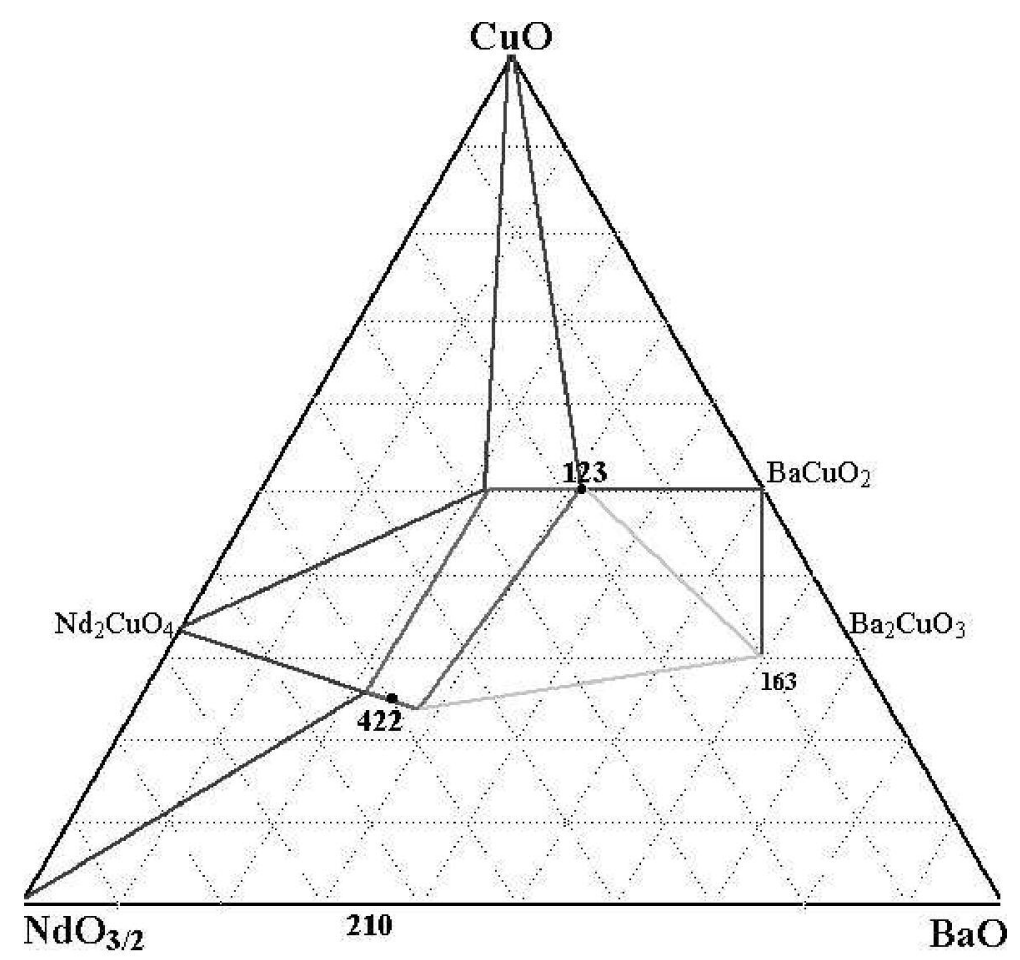

Fig. 2

$191 \times 272 \mathrm{~mm}(600 \times 600 \mathrm{DPI})$ 


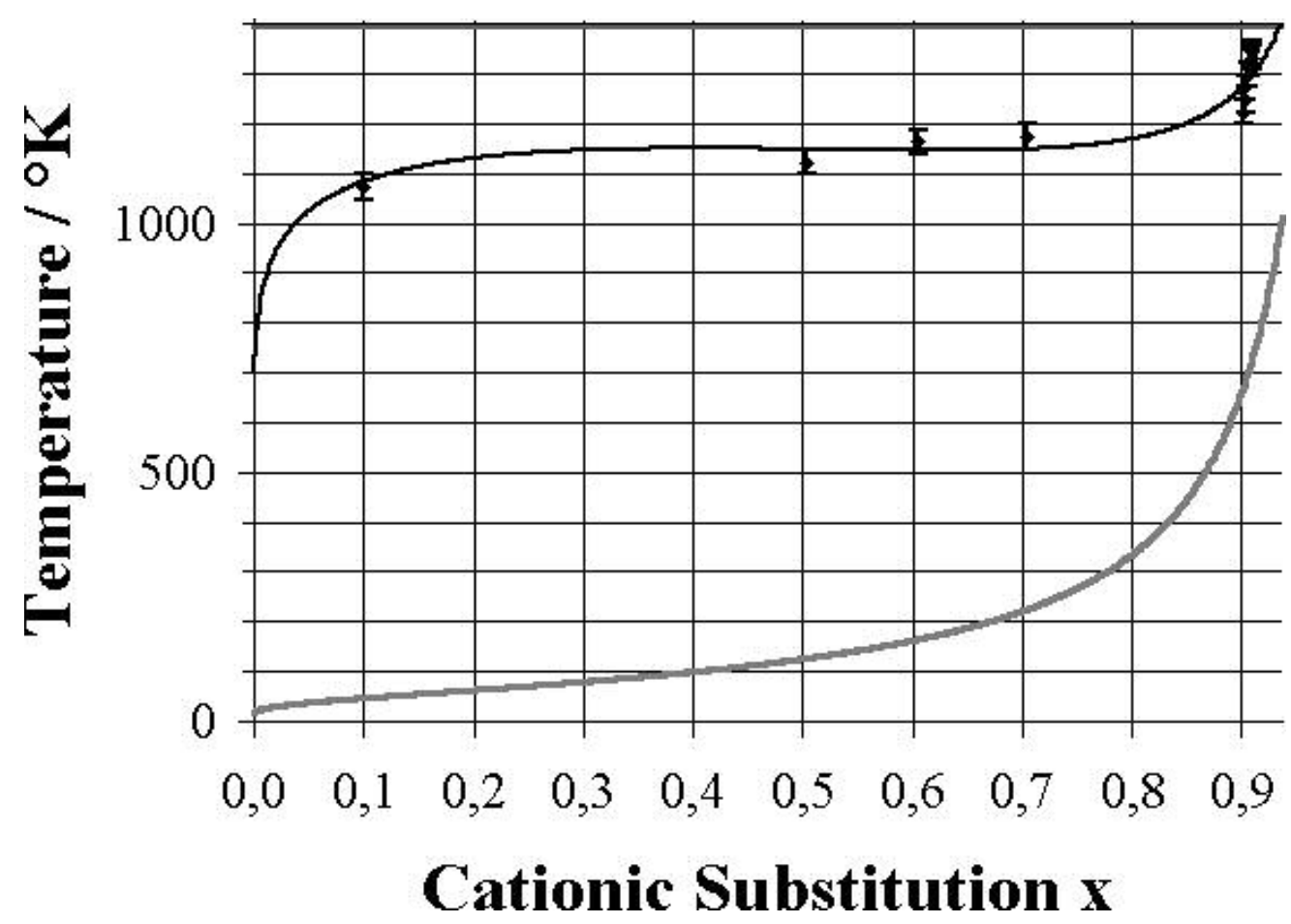

Fig.3

$203 \times 144 \mathrm{~mm}(72 \times 72$ DPI $)$ 


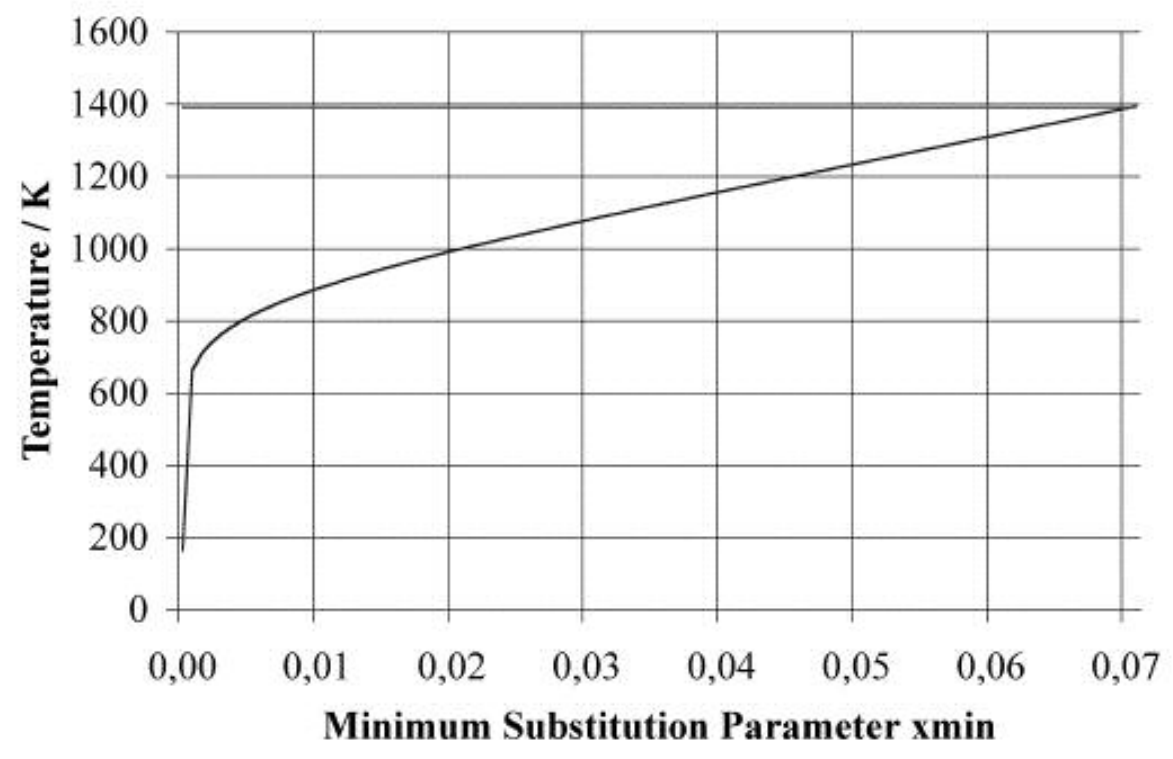

Fig.4

$203 \times 122 \mathrm{~mm}(72 \times 72$ DPI $)$ 